\title{
A Quantitative Approach for Developing Serious Games for Aptitude and Trait Assessment
}

\author{
Brenton M. Wiernik ${ }^{1[0000-0001-9560-6336]}$ and Michael D. Coovert ${ }^{1[0000-0003-3515-6753]}$ \\ ${ }^{1}$ University of South Florida, Tampa, FL 33620 \\ brenton@wiernik.org
}

\begin{abstract}
We describe a development process for serious games to create psychometrically rigorous measures of individual aptitudes (abilities, skills) and traits (habits, tendencies, behaviors). We begin with a discussion of serious games and how they can instantiate appropriate cognitive states for relevant aptitudes and traits to manifest. This can have numerous advantages over traditional assessment modalities. We then describe the iterative approach to aptitude and trait measurement that emphasizes (1) careful definition and specification of the traits and aptitudes to be measured, (2) rigorous assessment of reliability and validity, and (3) revision of gameplay elements and metrics to improve measurement properties.
\end{abstract}

Keywords: assessment, validity, factor analysis, personality, cognitive ability.

\section{Overview}

Selecting employees with necessary competencies to perform their jobs is critical to ensure workforces are productive and able to meet organizational goals, especially for cognitively demanding careers, such as software development, data science, and cybersecurity. Identifying the most capable employees requires precise, valid assessment of jobrelevant aptitudes and traits that contribute to employees' performance. In this proposal, we lay the groundwork for a philosophy of measurement for assessing aptitudes and traits using serious games. We begin with a discussion of serious games and their potential advantages and disadvantages compared with traditional assessment modalities. We emphasize the potential power of serious games to create an experience for the player with high psychological fidelity to the future work environment [1]. This allows players' ingame behavior to reflect their standing on job-relevant traits and aptitudes and to predict future on-the-job performance. Next, we describe a development sequence to ensure that serious game-based measures are psychometrically sound before applying them in operations. This sequence involves careful definition and specification of the traits and aptitudes to be measured, assessment of measures' reliability and validity following traditional psychometric standards [2], and iterative revision and refinement of gameplayrelated metrics to improve psychometric properties. 


\subsection{Serious Games}

Serious games are an evolving tool in organizations. Most current applications focus on education and training $[3,4]$. An emerging area of interest seeks to also apply games as part of a technologically enhanced employee selection process. Here, the goal is to use information collected during gameplay to assess a player's standing on job-relevant aptitudes and traits to determine their suitability for specific work roles. Below, we review existing types of serious games, consider common features of serious games that may prove useful for personnel assessment, and consider advantages and disadvantages of serious games relative to traditional assessment modalities.

\subsection{Existing Classifications of Serious Games}

We can consider the potential scope of serious game-based assessment by examining existing serious game taxonomies. A widely-used taxonomy describes six major categories of competencies (aptitudes and traits) that serious games have been designed to develop or assess $[5,6]$ :

1. Cognitive and perceptual competencies (e.g., problem-solving, planning)

2. Sensory-motor competencies (e.g., reaction time, eye-hand coordination)

3. Emotional and volitional competencies (e.g., stress control, endurance)

4. Personal and attitudinal competencies (e.g., self-efficacy, interest)

5. Social competencies (e.g., cooperation, communication)

6. Domain-specific competencies (e.g., media knowledge, job knowledge)

These categories of competencies (aptitudes and traits) are similar to major categories of competencies identified in job analysis and competency modeling practice in industrial psychology [7]. Ludoscience [8] has developed another serious game classification system that provides a directory of available games with summaries and searchable keywords. One may also examine the Serious Games Association directory [9].

\subsection{Psychological Fidelity as a Strength of Game-Based Assessment}

The key potential strength of serious games as a modality for employee assessment is their ability to create an immersive experience with high psychological fidelity to the onthe-job work environment $[9,10]$. That is, the game can place the player into cognitive and emotional states that resemble those experienced on the job [11]. All the elements of game design can be leveraged to evoke key work-relevant competencies, permitting each player's relative standings on key aptitudes and traits to manifest themselves through gameplay. These elements include (1) mechanics - procedures and rules of the game; (2) theme and story - the context and sequence of events that unfold in the game; (3) aesthetics - how the game looks, sounds, and feels; and (4) the technological media through which players interact with the game (e.g., displays, keyboards, physical apparatuses, or augmented reality devices [12] (p. 41). For example, strong themes and well-designed aesthetics can evoke specific emotional states. Using time limits, a fast pace, low lighting, or emotional or startling imagery or sounds can induce feelings of stress, urgency, or 
confusion. Assessing players' responses to such situations can be useful for many occupations, such as public safety and medicine. Similarly, game mechanics might be designed so that a team of players must cooperate to achieve shared goals; such a design could be used to assess individuals' cooperativeness or to assess a teams' communication skills or shared mental models. The sequence of game experiences over time can also be used as part of assessment. For example, games can be designed so that gameplay becomes progressively harder, permitting assessment of players' ability to acquire new skills or adapt to changing circumstances.

\subsection{Advantages and Disadvantages of Assessment via Serious Games}

Traditional aptitude assessments typically take the form of text-based questions and response options presented to applicants on paper or a computer screen. Questions may assess general capabilities (e.g., reasoning ability) or domain-specific knowledge. Their target aptitudes are obvious to applicants, and their presentation is not contextualized for a specific goal other than assessment. Traditional trait assessments typically involve selfreport responses to questions about typical behaviors in various settings. Trait assessment relies on applicants' descriptions of their own behavior, rather than observable performance. Alternative assessment modalities, such as work samples, simulations, and situational judgment tests, aim to provide higher fidelity assessment of job-relevant competencies [11]. Serious games can be understood as a new approach in this tradition of highfidelity, performance-based assessment.

Compared to traditional assessment modalities, serious games may have several advantages for assessing cognitive workers. First, serious games may elicit more favorable reactions from assessed applicants. Although traditional aptitude measures (e.g., general cognitive ability or reasoning measures) are widely applied in employment contexts, advanced employees may feel that such measures are inappropriate or insulting for employees of their level $[13,14]$. Similarly, high-level applicants may respond negatively to traditional self-report trait measures, for example, believing that such measures are too easily "faked" or otherwise are unable to accurately predict future performance [15]. Although such beliefs are likely inaccurate [16], applicants may respond better to serious game assessments due to their greater job fidelity and higher production values. Second, related to the first point, serious games may be more engaging for applicants and produce greater test-taker motivation [17]. Traditional cognitive assessments may be perceived as boring or uninteresting; even if applicants are extrinsically motivated to perform well in order to be selected for a position, uninteresting tasks may reduce intrinsic motivation, preventing applicants from performing at maximal levels and potentially contaminating assessments with extraneous capabilities (e.g., attentional control). By providing an assessment experience that is intrinsically motivating, serious games may help to elicit maximal performance during applicant assessment. Third, compared to traditional selfreport trait measures, serious game assessments may be less prone to impression management [18] (but cf. [16]). Applicants may be less able to discern the targeted traits in a serious game compared to traditional assessments, so they are less able to modify their responses to inflate their expected scores. Fourth, serious games may be able to assess multiple aptitudes and traits at one time by using a variety of gameplay metrics recorded 
concurrently. This can potentially reduce assessment time compared to traditional multiscale assessment batteries.

Despite these potential advantages, serious games also have several potential disadvantages that must be overcome to ensure reliable and valid assessment. First, it may be difficult to develop gameplay metrics that can function as indicators of targeted aptitudes and traits without contamination by undesired characteristics (e.g., video game familiarity, psychomotor skills). Second, if multiple traits or aptitudes are assessed using performance on the same gameplay tasks, these scores may show a lack of discriminant validity (unique variance for each assessment), even if they are scored using distinct gameplay metrics. For example, many performance-based metrics (e.g., achievement of game objectives, resource management, gameplay speed) are likely to be influenced by general cognitive ability and overall game skill, leading to substantial correlations among these measures. Third, some traits or aptitudes may be difficult to assess within the context of a game and may be better assessed through traditional methods. For example, Conscientiousness-related traits (responsibility, dependability, achievement striving) refer primarily to behaviors exhibited over the long term. Valid measures of these characteristics may be difficult to obtain during a short gameplay period; more accurate assessment could likely be realized using self-report, other-report, or other methods than can refer to longterm typical behavioral patterns. Fourth, relatedly, some traits may take longer to assess reliably using a serious game than using a traditional method. In designing serious games for personnel assessment, developers must take caution to avoid these disadvantages and ensure that the serious game provides real benefits over traditional alternative assessment modalities.

\section{Development Sequence for Game-Based Assessments}

\subsection{Serious Games as a Means to an End}

When designing a serious game for personnel assessment, it is critical to remember that the purpose of the game is to obtain reliable, predictive assessments of applicant traits and aptitudes - the game is only a means to this end. It does not matter how clean, slick, expensive, or useful the game appears; if it cannot reliably assess the targeted competencies, the game will be unable to provide useful information to aid organizations' decision making. This purpose must guide all game design, development, validation, and deployment decisions. This purpose of personnel assessment games - to accurately measure job-relevant traits, not merely entertain —is the game's "characterizing goal" [9] (p. 3). Achieving this goal requires not only quality game design, but clear specification of the assessment purposes and rigorous evidence of psychometric validity.

\subsection{Assessment Purpose: Construct Definition and Specification}

Development of serious game assessments must follow rigorous procedures to ensure reliability, validity, utility, and fairness [2]. The first step of this process is to clearly define the traits and aptitudes ("constructs") that the game will measure and to specify 
how these characteristics are linked with on-the-job performance (e.g., what level of an ability is relevant for the job? under what circumstances must employees demonstrate a trait?). These decisions should be informed by job analysis, a process through which key work tasks are described and the necessary aptitudes and traits to perform them are delineated [19]. The constructs to be assessed must be carefully chosen so they are (1) necessary for effective job performance, (2) amenable to assessment using gameplay performance, and (3) not already well-assessed using existing measures and methods.

\subsection{Selecting Game Assessment Metrics}

After specifying the aptitudes and skills to be measured, the next step is to select the specific metrics that will be used to quantify these competences. Assessment in a serious game typically occurs via three primary methods - (1) direct assessment by the game engine during gameplay, (2) ratings by trained observers, or (3) player responses to questions during or after play $[10,20]$. Some constructs, such as decision-making ability, may be amenable to direct assessment by the game engine (e.g., by achievement of game objectives), whereas others (e.g., team cohesion, emotional states) may be better-assessed using observer ratings or player responses to post-game reflection questions.

When selecting performance metrics to monitor as assessments of target constructs, it is important to align assessment granularity with the level needed to inform decisions. Human performance occurs at multiple levels of specificity-for example, neurological, biomechanical, cognitive, and social. The same phenomenon can be described simultaneously at multiple levels. For example, in a resource management game, performance could be assessed at the biomechanical level using a stream of individual clicks and movements. It could also be assessed more abstractly at the cognitive level by capturing the aggregate number of errors and efficiency of the player's allocation decisions. In many cases, focusing assessment at the more abstract cognitive and social levels will produce more actionable performance information than minute neurological or biomechanical information [21]. For a summary, see [22] (pp. 275-277).

In designing serious game assessments, developers must conceive of observable performance behaviors and outcomes as indicators of the underlying latent traits and aptitudes they wish to assess [10]. Game performance itself is not of interest; performance metrics are only valuable to the degree that they can be used to infer players' standing on targeted job-relevant traits and aptitudes. Directly observable behaviors and responses may be driven by multiple traits and aptitudes; game designers must carefully consider the myriad of factors that may contribute to performance behaviors and select metrics (or apply analytic models) to isolate relevant trait and aptitude information. Gameplay must also be designed to avoid evoking unintended responses. For example, game difficulty should be increased at an appropriate rate to engage and challenge players without inducing frustration or anxiety.

Performance evolves over the course of gameplay, and changes over time may impact the validity of assessments. Considering the stage of gameplay is critical for serious game assessment. For example, early in gameplay, players may need time to become familiar with the game controls and objectives. Early performance may thus more reflect factors such as prior gaming experience (i.e., "game wise-ness"), rather than the constructs of 
interest. To counter such effects, developers might consider providing a warmup period for the player to practice and become comfortable with play before beginning scored assessment. The constructs driving successful game performance may also change over the course of play. For example, in a team simulation, early team-level performance might capture team trust-willingness to work with and rely on other people. Later in play, when players are more comfortable with each other, successful performance may be more driven by effective communication and teamwork skills.

Assessment metrics might also consider players' dynamic performance over time. Does performance improve over time? Is it consistent or highly variable? How does performance vary across different situations, task types, stimuli, or difficulty levels? These qualities are often difficult to assess in the staffing process; thus, a game's ability to capture performance dynamics is a potential advantage over traditional modalities.

\subsection{Measurement and Modeling}

This section considers the issue of measurement - how observable game performance metrics are used to infer underlying trait and aptitude levels. After identifying candidate metrics to assess each construct, formal models must be built and evaluated to convert raw performance metrics into scores for targeted constructs. A variety of psychometric and statistical models are available to model latent traits and aptitudes. We review three common models that may be particularly useful for serious game-based assessment.

\section{Common Factor Model.}

A common factor model specifies that each observed indicator comprises two parts(1) variance shared with other indicators due to a common factor, taken to represent the construct of interest and (2) variance unique to each indicator, usually taken to represent measurement error. For example, several game objectives may be designed to capture decision-making ability. Success/failure for each objective can be modeled as indicators (a la test items) of a latent Decision-Making factor. We show such a model in Figure 1 with standard RAM notation from structural equation modeling. Ellipses are latent constructs, boxes are observed/measured variables, single-headed arrows are factor loadings/regression paths, and double-headed arrows are variances or residual/error variances. Here, $G$ is a common factor purported to underlie performance on the six observed indicators $M 1-M 6$. The $\lambda$ values are factor loadings - regression coefficients predicting each observed variable using the latent factor.

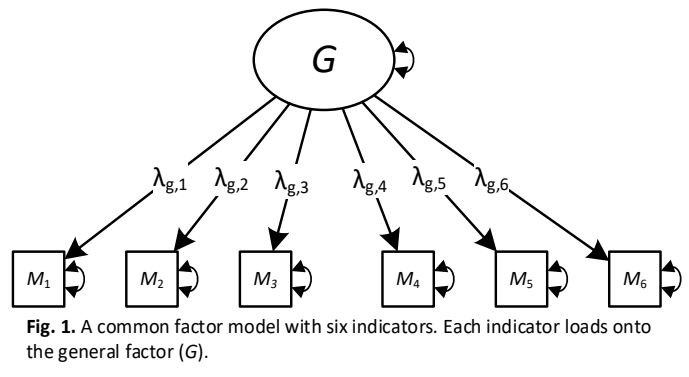

A common factor model is appropriate when a weighted composite of indicators can well-represent the construct. For an overall score to be interpretable, the measure should be unidimensional or at least possess a strong general factor that captures most of the variance in observed indicators [23, 24]. For guidelines on common factor models, see [25, 26]. 


\section{Bifactor Model.}

A bifactor model (Figure 2) specifies that each observed indicator comprises three parts - (1) variance shared with all other indicators due to a common general factor, $G$, (2) variance shared with a subset of other indicators due to "group factors", $S$, and (3) variance unique to each indicator (again interpreted as measurement error) [27].

Bifactor models are useful when indicators violate the unidimensional common factor model's strict conditional independence assumption - that indicators are independent after accounting for a single common factor [27]. The general and group factors in a bifactor model could all be substantively meaningful (e.g., a broad trait and sub-traits), or one or the other could be interpreted as a measurement artefact. For example, a bifactor model may be useful in serious game assessment to increase discriminant validity (reduce collinearity) between scores for different constructs. Performance-based metrics based on the same gameplay sequence are likely to be highly correlated. These correlations may be substantive (e.g., overall skill or aptitude), but they may also reflect shared method

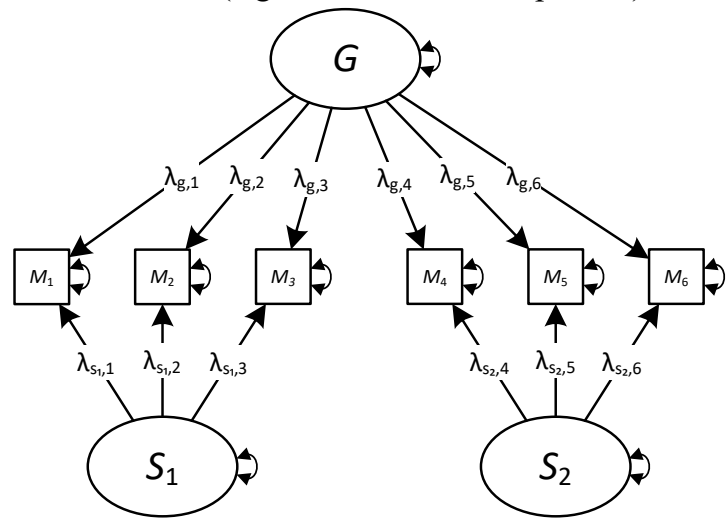

Fig. 2. A bifactor model with six indicators. Each indicator loads onto the general factor $(G)$ and onto one group factor $\left(S_{1}\right.$ or $\left.S_{2}\right)$. artefacts. Removing this common variance using a bifactor model can help to produce more distinct scores for specific aptitudes, permitting differential assessment and comparison of players across multiple constructs. Caution is warranted, however; if the general factor is strong, group factor scores after removing it may be very unreliable (imprecisely estimated) $[24,28]$. For guidance on bifactor models, see [27, 29].

\section{Growth Curve and Latent Change Score Models.}

A third useful model is a growth curve model (Figure 3), as well as the related latent change score model [30]. In a latent growth curve model, a construct is assessed repeatedly over time, and changes in the scores are used to model growth trajectories for each person. Each person's growth is modeled using an intercept factor, with all loadings fixed

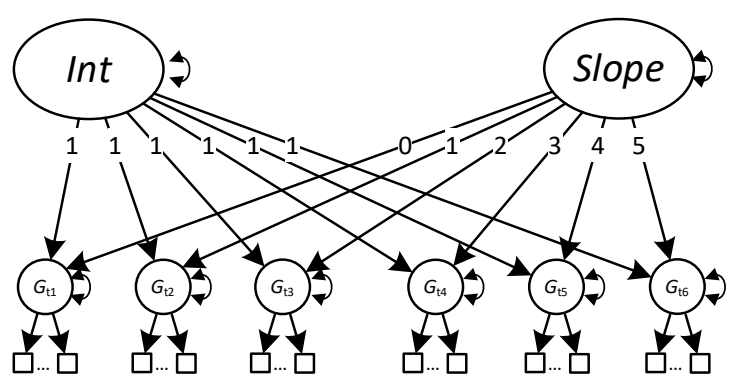

Fig. 3. A latent growth curve model with six time points $\left(G_{\mathrm{t} 1}-G_{\mathrm{t} 2}\right)$, an intercept (Int), and a linear growth term (Slope). Each timepoint is a common factor model with observed indicators ( $\square$; full measurement models not shown). to 1 , and one or more slope factors, with loadings fixed to values based on the amount of time elapsed or number of measurements taken. Different types of growth trajectories can be modeled (e.g., linear, quadratic). Growth curve models are useful for assessing development or change. For example, they could be used to quantify how quickly 
players learn a skill. Coovert et al. [31] used growth curve models to assess teammate trust development during a cooperative serious game. For guidance on growth curve models, see [32].

Typically, the number of candidate metrics will be relatively small, as they will be specifically designed to assess specific constructs. If a large number of metrics are being considered, other modeling approaches, such as regularized regression or genetic algorithms, may also be useful [33].

\section{Reliability and Measurement Precision.}

Trait and aptitude scores derived from serious game-based assessments must be estimated with sufficient precision (1) to discern applicants' specific competency levels and (2) to discriminate between applicants with different competency levels. This means that standard errors for individual scores must be small. This is the concept of measure reliability [2]. If the estimated models yield scores with large standard errors, assessments should be revised until standard errors are small enough to meet decision-making needs. This can include revising metrics to be higher quality or more reliable or adding additional metrics to the scoring model. To predict future job performance, scores must also have high test-retest reliability (stability over time) [2].

\subsection{Validation}

After designing a serious game, choosing performance metrics, and modeling these metrics to estimate reliable scores, further analyses are needed to evaluate the validity of the scores - whether they can be accurately interpreted as reflecting individuals' standing on the intended traits or aptitudes, as well as whether they accurately predict on-the-job performance [2]. Several types of validity evidence of must be gathered before operational use of a serious game-based assessment can be justified. The first is construct validity evidence. The serious game-based scores should be shown to correlate with scores on other measures of the same or similar constructs (convergent validity; e.g., problem-solving game scores should correlate with traditional deductive and inductive reasoning assessments). They should also show weak correlations with measures of other constructs (discriminant validity; e.g., a measure of stress tolerance should be unrelated to reasoning ability, whether assessed using traditional modalities or game-based metrics). The second type of validity evidence is criterion-related validity evidence. Serious game-based scores should be shown to correlate with relevant job performance behaviors and outcomes (e.g., a measure of learning speed should predict training performance; a measure of cooperativeness should predict teamwork). Finally, assessments should be shown to function equivalently (show measurement and predictive invariance) across groups, such as gender, racial, age, and language groups.

Collecting data to show these types of validity evidence helps to ensure that scores can be accurately interpreted as reflecting the intended competencies and to rule out alternative interpretations (e.g., that scores merely reflect game-playing skill, gender bias, or noise). The role of sound psychometric analysis in this work cannot be overstated; without accurate and rigorous psychometric evidence supporting proposed interpretations of calculated scores, it is impossible know whether assessment results can usefully inform 
decision making practice. Unless robust reliability and validity evidence is presented, there is no way to know whether a serious game is useful or merely a pleasant diversion.

\subsection{Iteration and Refinement}

Developing a serious game that can reliably and validly assess job-relevant aptitudes and traits is a challenging process. In most cases, many rounds of revision and refinement will be needed before an assessment meets psychometric standards for operational use. Game performance metrics that were initially expected to reliably indicate a specific construct may prove unreliable or unrelated to existing measures of these constructs. Even reliable and valid metrics may show insufficient variance to discriminate individuals with different construct levels (e.g., if game tasks are too easy or challenging for the job applicant population). Serious game-based measures, just like traditional psychometric assessments, require extensive testing and revision before measures can be validly applied in practice. Even the game design itself (separate from design of game-based assessments - controls, audiovisual assets, difficulty, etc.) requires extensive playtesting and revision to ensure the game is playable and engaging for players.

Given the need for extensive testing and revision, serious game developers should adopt an iterative or spiral development model [12] (p. 83), beginning with an initial prototype followed by testing, revision, further testing, further revision, and so forth. This process continues until acceptable levels of playability and assessment reliability and validity are achieved.

\section{Summary}

Developing psychometrically reliable and valid measures of aptitudes and traits is critical to inform selection of employees, especially for cognitively demanding jobs. Serious games present an exciting new technology that may address important limitations of traditional assessment modalities. Attention to the psychometric properties of assessments during serious game development is paramount. Designing a serious game that creates a terrific play experience is of no benefit if it fails to deliver an accurate assessment the targeted aptitudes and traits. In this paper, we described a framework for developing and iteratively evaluating and refining game-based assessments to ensure the resulting assessment products can meaningfully inform personnel decision-making.

\section{$4 \quad$ References}

1. Kozlowski, S.W.J., DeShon, R.P.: A psychological fidelity approach to simulationbased training: theory, research and principles. In: Schiflett, S.G., Elliott, L.R., Salas, E., and Coovert, M.D. (eds.) Scaled worlds: development, validation and applications. Routledge, London, UK (2017). https://doi.org/10/c7n3

2. Society for Industrial and Organizational Psychology, American Psychological Association: Principles for the validation and use of personnel selection procedures 
(Fifth edition) (2018). https://www.apa.org/ed/accreditation/about/policies/personnel-selection-procedures.pdf

3. Ford, J.K., Meyer, T.: Advances in training technology: meeting the workplace challenges of talent development, deep specialization, and collaborative learning. In: Coovert, M.D. and Thompson, L.F. (eds.) The psychology of workplace technology. Routledge, New York, NY (2013). https://doi.org/10/c7n2

4. Long, D.T., Mulch, C.M.: Interactive wargaming cyberwar: 2025, (2017). https://apps.dtic.mil/docs/citations/AD1053350

5. Wiemeyer, J., Hardy, S.: Serious games and motor learning: concepts, evidence, technology. In: Bredl, B. and Bösche, W. (eds.) Serious games and virtual worlds in education, professional development, and healthcare. pp. 197-220. IGI Global, Hershey, PA (2013). https://doi.org/10/gf392r

6. Wiemeyer, J., Kliem, A.: Serious games in prevention and rehabilitation-a new panacea for elderly people? Eur. Rev. Aging Phys. Act. 9, 41-50 (2012). https://doi.org/10/bfxfmn

7. The O*NET® Content Model. https://www.onetcenter.org/content.html

8. Ludoscience: A collaborative classification of serious games. http://serious.gameclassification.com/

9. Dörner, R., Göbel, S., Effelsberg, W., Wiemeyer, J. eds: Serious games: foundations, concepts and practice Springer, Cham, Switzerland (2016). https://doi.org/10/c7n4

10. Coovert, M.D., Winner, J., Bennett, W.: Construct development and validation in game-based research. Simul. Gaming. 48, 236-248 (2017). https://doi.org/10/gd892c

11. Lievens, F., Patterson, F.: The validity and incremental validity of knowledge tests, low-fidelity simulations, and high-fidelity simulations for predicting job performance in advanced-level high-stakes selection. J. Appl. Psychol. 96, 927-940 (2011). https://doi.org/10/dtv6hr

12. Schell, J.: The art of game design: a book of lenses CRC Press, Boca Raton, FL (2008). https://doi.org/10/c7n5

13. Anderson, N., Salgado, J.F., Hülsheger, U.R.: Applicant reactions in selection: comprehensive meta-analysis into reaction generalization versus situational specificity. Int. J. Sel. Assess. 18, 291-304 (2010). https://doi.org/10/dkgzm8

14. Anderson, N.: Applicant and recruiter reactions to new technology in selection: a critical review and agenda for future research. Int. J. Sel. Assess. 11, 121-136 (2003). https://doi.org/10/b2mqtk

15. Gilliland, S.W.: Fairness from the applicant's perspective: reactions to employee selection procedures. Int. J. Sel. Assess. 3, 11-18 (1995). https://doi.org/10/fpfm6t

16. Ones, D.S., Viswesvaran, C., Reiss, A.D.: Role of social desirability in personality testing for personnel selection: the red herring. J. Appl. Psychol. 81, 660-679 (1996). https://doi.org/10/c7kgg9

17. Eklöf, H.: Skill and will: test-taking motivation and assessment quality. Assess. Educ. Princ. Policy Pract. 17, 345-356 (2010). https://doi.org/10/fkj3dp

18. McFarland, L.A., Yun, G.J., Harold, C.M., Viera, L., Moore, L.G.: An examination of impression management use and effectiveness across assessment center exercises: 
the role of competency demands. Pers. Psychol. 58, 949-980 (2005). https://doi.org/10/dfqs3k

19. Wilson, M.A. ed: The handbook of work analysis: methods, systems, applications and science of work measurement in organizations Routledge, New York (2013). https://doi.org/10.4324/9780203136324

20. Coovert, M.D., Winner, J., Bennett, W., Jr., Howard, D.J.: Serious games are a serious tool for team research. Int. J. Serious Games. 4, (2017). https://doi.org/10/gfs 7 tw

21. Campbell, J.P., Wiernik, B.M.: The modeling and assessment of work performance. Annu. Rev. Organ. Psychol. Organ. Behav. 2, 47-74 (2015). https://doi.org/10/bc4k

22. Wiemeyer, J., Kickmeier-Rust, M., Steiner, C.M.: Performance assessment in serious games. In: Dörner, R., Göbel, S., Effelsberg, W., and Wiemeyer, J. (eds.) Serious games: foundations, concepts and practice. pp. 273-302. Springer, Cham, Switzerland (2016). https://doi.org/10/c7n7

23. Nandakumar, R.: Assessing essential unidimensionality of real data. Appl. Psychol. Meas. 17, 29-38 (1993). https://doi.org/10/cb827h

24. Gignac, G.E., Watkins, M.W.: Bifactor modeling and the estimation of model-based reliability in the WAIS-IV. Multivar. Behav. Res. 48, 639-662 (2013). https://doi.org/10/gckfjn

25. Coovert, M.D., McNelis, K.: Determining the number of common factors in factor analysis: a review and program. Educ. Psychol. Meas. 48, 687-692 (1988). https://doi.org/10/c4n67f

26. Comrey, A.L.: A first course in factor analysis (Second edition) Psychology Press, New York, NY (2013). https://doi.org/10/c7n8

27. Reise, S.P.: The rediscovery of bifactor measurement models. Multivar. Behav. Res. 47, 667-696 (2012). https://doi.org/10/gckf67

28. Wiernik, B.M., Wilmot, M.P., Kostal, J.W.: How data analysis can dominate interpretations of dominant general factors. Ind. Organ. Psychol. 8, 438-445 (2015). https://doi.org/10/895

29. Giordano, C.A., Waller, N.G.: Recovering bifactor models: a comparison of seven methods. Psychol. Methods. (2019). https://doi.org/10.1037/met0000227

30. McArdle, J.J.: Latent variable modeling of differences and changes with longitudinal data. Annu. Rev. Psychol. 60, 577-605 (2009). https://doi.org/10/dhxt7h

31. Coovert, M., Miller, E., Bennett, W., Jr.: Assessing trust and effectiveness in virtual teams: latent growth curve and latent change score models. Soc. Sci. 6, 87 (2017). https://doi.org/10/gf3922

32. Bollen, K.A., Curran, P.J.: Latent curve models: a structural equation perspective Wiley, Hoboken, NJ (2005). https://doi.org/10/bn3pwk

33. Kuhn, M., Johnson, K.: Applied predictive modeling Springer, New York, NY (2013). https://doi.org/10/c432 